\title{
Solvatochromic effect in absorption and emission spectra of star-shaped bipolar derivatives of 1,3,5-triazine and carbazole. A time-dependent density functional study
}

\author{
Gleb V. Baryshnikov ${ }^{1,2} \cdot$ Sergey V. Bondarchuk ${ }^{2} \cdot$ Valentina A. Minaeva ${ }^{2}$. \\ Hans Ågren ${ }^{1}$ - Boris F. Minaev ${ }^{1,2}$
}

Received: 11 November 2016/Accepted: 13 January 2017 / Published online: 4 February 2017

(C) The Author(s) 2017. This article is published with open access at Springerlink.com

\begin{abstract}
A series of three star-shaped compounds containing both donor (carbazole) and acceptor (2,4,6-triphenyl-1,3,5triazine) moieties linked through various linking bridges was studied theoretically at the linear response TD-DFT level of theory to describe their absorption and fluorescence spectra. The concept of a localized charge-transfer excited state has been applied successfully to explain the observed strong solvatochromic effect in the emission spectra of the studied molecules, which can be utilized for the fabrication of color tunable solution-processable OLEDs. The concept is in particularly applicable to donor-acceptor species with a $C_{3}$ symmetry point group where the static dipole moment changes dramatically upon electronic excitation. An important peculiarity of the studied molecules is that they are characterized by non-zero values of the HOMO and LUMO orbitals in the same common part of molecular space that provides a large electric dipole transition moment for both light absorption and emission.
\end{abstract}

Keywords Star-shaped compounds · OLEDs · TDDFT · Solvatochromic effect $\cdot$ Dipole moment

Electronic supplementary material The online version of this article (doi:10.1007/s00894-017-3234-y) contains supplementary material, which is available to authorized users.

Gleb V. Baryshnikov

glebchem@rambler.ru

1 Division of Theoretical Chemistry and Biology, School of Biotechnology, KTH Royal Institute of Technology, 10691 Stockholm, Sweden

2 Department of Chemistry and Nanomaterials Science, Bogdan Khmelnitsky Cherkasy National University, blvd. Shevchenko 81, 18031 Cherkasy, Ukraine

\section{Introduction}

In recent years, star-shaped organic materials have attracted a great deal of attention due to their promising applications in organic light-emitting devices (OLEDs) [1-6]. One of the main challenges in this field is the realization of ambipolar transporting properties within single-type molecules, together with the strong photoluminescence that allows improvement of the luminance characteristics of OLEDs [5]. Another important feature of star-shaped organic luminophores is that they tend to form exciplexes with a wide range of organic materials, which is very useful for the emission-color tuning ability of OLEDs [7-9].

The general strategy to create ambipolar star-shaped emitters is to combine donor (D) and acceptor (A) fragments within the same molecule [5, 6], which (1) facilitates injection and transport properties of both charge-carriers-holes and electrons, and (2) activates intermolecular charge-transfer (CT) excited states in the photoluminescence spectra. Such CT states usually are the lowest-lying excited states of the starshaped molecules, i.e., they are responsible for the fluorescence process. Moreover, the nonzero values of the HOMO and LUMO wave-functions in the same common part of the molecular space is a very important property of efficient starshaped emitters [10,11], providing a large electric dipole transition moment for both light absorption and emission [12].

It is well-known that D-A molecules usually demonstrate solvent-dependent behavior in their absorption spectra due to the high polarization of the ground-state molecular structure [12-17]. Strong positive solvatochromism in absorption spectra is observed frequently for molecules with a $\pi \pi^{*}$ nature of band-productive electronic state. The absolute value of the red shift depends, usually linearly, on the solvent polarity (the higher the solvent polarity, the stronger the red shift) [6]. This is because the more polar solvent species polarizes 
molecules with a higher static dipole moment more strongly (particularly for D-A systems). But, in the case of symmetrical star-shaped molecules (3D-A, for example), the static dipole moment of the whole system is equal to zero because of the $C_{3}$ symmetry point group restriction. Therefore, these systems should not demonstrate strong solvatochromism in the absorption spectra. However, if the excited state of the star-shaped molecule corresponds to the local CT $\pi \pi^{*}$-state, the structure of the excited-state geometry should be significantly distorted and the dipole moment of the excited state should differ markedly from zero. This means that clear solvatochromic behavior should be observed in the emission (fluorescence) spectra of D-A star-shaped molecules with local CT excited states rather than in absorption spectra where the vertical excitation prevails. In this way we can vary the emission color of the starshaped compound in various solvents using the same excitation energy, which is very useful for applications of $C_{3}$ symmetry point group in photovoltaic cells, OLEDs and bioimaging technologies [6].

In the present work, we focused on the three recently synthesized star-shaped compounds containing both $\mathrm{D}$ (carbazole) and A (2,4,6-triphenyl-1,3,5-triazine) moieties connected through various linking bridges [6]. We describe the results of quantum-chemical calculations carried out in order to study the "structure-optical properties relationship" of these D-A materials. Such compounds demonstrate clear solvent-dependent fluorescence, but the solvent effect is less observable in the absorption spectra. We think that the selected star-shaped compounds are really good candidates to prove the theory of local CT excited states in organic fluorofores of the $C_{3}$ symmetry point group.

\section{Computational details}

The calculations presented in this paper were performed in terms of density functional theory (DFT) using the Gaussian09 suite of programs $[18,19]$. Geometry optimizations were carried out by the hybrid exchange-correlation functional B3LYP $[20,21]$ with the Pople's split-valence basis set (almost double- $\zeta$ in the valence shell, $6-31 \mathrm{G})$ and addition of polarization $(\mathrm{d}, \mathrm{p})$ functions $[22,23]$. The optimized structures were checked for absence of imaginary frequencies in the vibrational spectra, and the geometries obtained were justified as global minima.

The UV-vis spectra were obtained in terms of timedependent density functional theory (TD-DFT) [24]. For this purpose, we applied the conventional B3LYP scheme as well as the modified B3LYP functional with the changed contribution of the exact Hartree-Fock exchange (HFE) part, which was increased up to $30 \%$. Furthermore, we used the PBE0 [25], mPW1PBE [26], CAM-B3LYP [27], BMK [28], $\omega B$ 97XD [29], and M062X [30] functionals. Polar media simulations were performed in terms of the polarizable continuum model (PCM) using integral equation formalism (IEFPCM) [31]. To define cavities, the universal force field (UFF) radii were used. The overlap index and minimum radius of the spheres were specified as 0.8 and $0.5 \AA$, respectively. We calculated the energies and oscillator strength values for 15 vertical electronic transition using the same $6-31 \mathrm{G}$ basis set as that used for geometry optimization. The fluorescence energies were computed, taking into account relaxation of the excited state geometry (TD-DFT optimization of the $\mathrm{S}_{1}$ state geometry), including the state-specific equilibrium solvation correction.

The electronic absorption spectra curves were fitted using the Gauss distribution function and a half-width of $3000 \mathrm{~cm}^{-1}$ with the SWizard 5.0 program package [32]. Molecular visualizations were performed with Chemcraft 1.6 [33].

\section{Results and discussion}

\section{Effect of exact HFE on UV-vis spectra prediction}

The optimized structures of the studied propeller-shaped bipolar derivatives of 1,3,5-triazine and carbazole, namely, 2,4,6-tris(4-(3-tert-butyl-carbazol-9-yl)phenyl)-1,3,5-triazine (TR1), 2,4,6-tris(4-(3,6-di-tert-butyl-carbazol-9-yl)phenyl)1,3,5-triazine (TR2) and 2,4,6-tris(4-((9-hexyl-carbazol-3yl)ethynyl)phenyl)-1,3,5-triazine (TR3) are illustrated in Fig. 1, and the calculated IR spectra (except of TR2) are listed in Table S1 and Fig. S1. The ground state of these structures belongs to the $C_{3}$ point group symmetry. As one can see in Table S1, the stationary points are characterized by the absence of imaginary frequencies, and are vibrationally stable.

To find the most appropriate functional for the absorption spectra calculation, we have performed a series of trials using hybrid functionals with different amounts of the exact HFE [34]. The results obtained are collected in Table 1. A strong correlation between the HFE (\%) and the $S_{0} \rightarrow S_{1}$ transition energy and intensity (oscillator strength) was found (Fig. S2). Except for the $\omega$ B97XD and CAM-B3LYP functionals, the following correlation coefficients were found: TR1 and TR2 $\left(R^{2}=0.9817\right)$, TR3 $\left(R^{2}=0.9866\right)$ for transition energy; TR1 and TR2 $\left(R^{2}=0.9995\right)$, TR3 $\left(R^{2}=0.9690\right)$ for oscillator strength. Note that the aforementioned correlations are presented for the hybrid functionals only. The rangeseparated functionals used in this study, namely, CAMB3LYP and $\omega \mathrm{B} 97 \mathrm{XD}$, were not involved in the correlation because they provide a different scheme for electron transition energy. As one can see in Table 1, the increased amount of HFE causes a rise in the transition energy. The rangeseparated functionals provide the same trend.

Herein, the custom-defined scheme (B3LYP-30) [18, 20, 21] provides the best fit with the experimental data $(3.19 \mathrm{eV})$ [17], and overestimates the transition energy by only $0.032 \mathrm{eV}$ 
Fig. 1 Structure of the species TR1-TR3 optimized by the DFT(B3LYP)/6-31G(d,p) method in the $n$-hexane [polarizable continuum model (PCM) using integral equation formalism (IEFPCM)] solvent
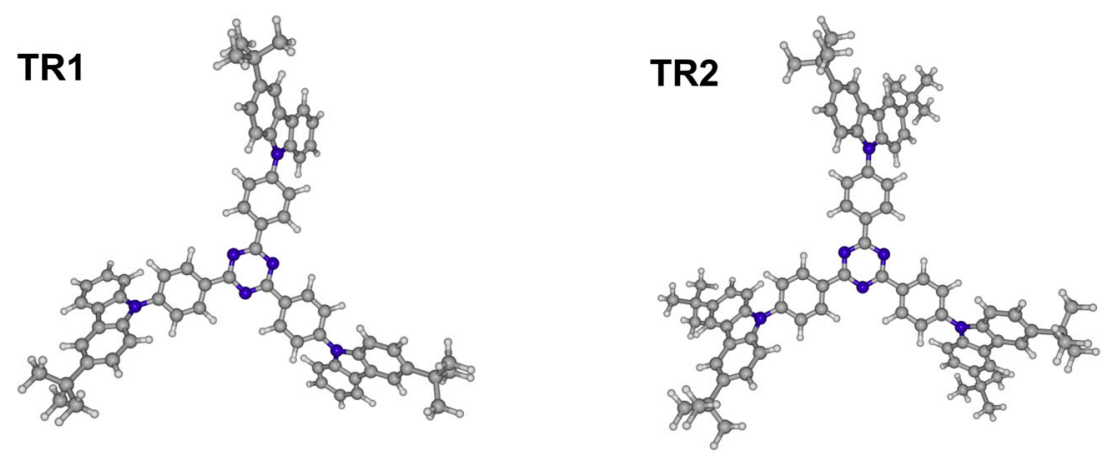

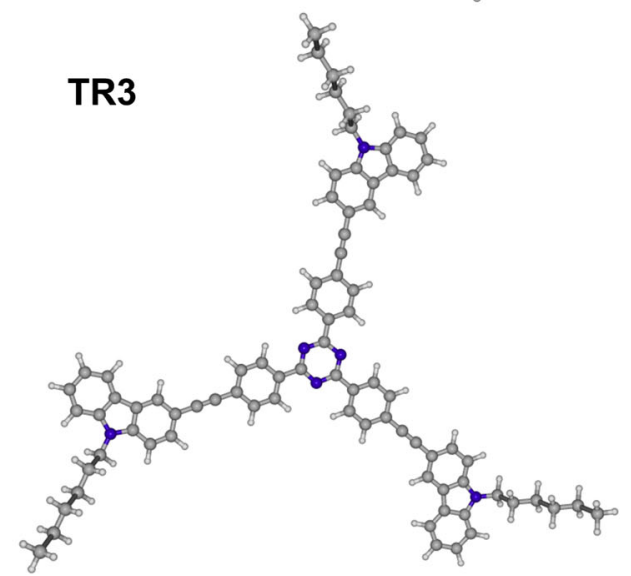

(Table 1). The regular B3LYP functional gave a strong underestimation of the transition energy (about $0.330 \mathrm{eV}$ ), which is a known limitation of this functional for the $\mathrm{CT}$ states [34]. All other hybrid functionals demonstrate the same underestimation trend (Table 1). The use of range-separated functionals like CAM-B3LYP usually ensures more adequate energies for the CT states, but in our case this type of functional strongly overestimated the fluorescence-responsible $S_{1}$ state energy (Table 1). This is due to the fact that the first $S_{1}$ state for the TR1-TR3 molecules combines the CT nature with local $\pi \rightarrow$ $\pi^{*}$-excitation. As can be seen from Fig. 2, the HOMO and
LUMO wave-functions are characterized by nonzero expansion coefficients in the same common part of the molecular space (phenyl ring nearest to the triazine core). This is a very important property of efficient light-harvesting end lightemissive materials. Such HOMO-LUMO “overlapping” provides a large electric dipole transition moment for light absorption and light emission processes $\left(\mathrm{S}_{0} \leftrightarrow \mathrm{S}_{1}\right)$ [12], and, therefore, all the studied molecules are characterized by high values of oscillator strength for $\mathrm{S}_{0} \rightarrow \mathrm{S}_{1}$ absorption as well as high fluorescence quantum yields for the $\mathrm{S}_{1} \rightarrow \mathrm{S}_{0}$ emission channel (about $80 \%$ in non-polar media). For this reason,
Table 1 Energy and intensity of the $S_{0} \rightarrow S_{1}$ transition [in hexane, the polarizable continuum model (PCM) using integral equation formalism (IEFPCM) model] in the absorption spectra of the species TR1-TR3 as functions of the Hartree-Fock exchange (HFE) contribution in the exchangecorrelation functional

\begin{tabular}{|c|c|c|c|c|c|c|c|c|c|c|}
\hline \multirow[t]{2}{*}{ Functional } & \multirow{2}{*}{$\begin{array}{l}\text { HFE } \\
(\%)^{\mathrm{a}}\end{array}$} & \multicolumn{3}{|l|}{ TR1 } & \multicolumn{3}{|l|}{ TR2 } & \multicolumn{3}{|l|}{ TR3 } \\
\hline & & $\begin{array}{l}\lambda \\
(\mathrm{nm})\end{array}$ & $\begin{array}{l}E \\
(\mathrm{eV})\end{array}$ & $f$ & $\begin{array}{l}\lambda \\
(\mathrm{nm})\end{array}$ & $\begin{array}{l}E \\
(\mathrm{eV})\end{array}$ & $f$ & $\begin{array}{l}\lambda \\
(\mathrm{nm})\end{array}$ & $\begin{array}{l}E \\
(\mathrm{eV})\end{array}$ & $f$ \\
\hline Exp. $^{b}$ & & 389 & 3.19 & & 397 & 3.12 & & 394 & 3.14 & \\
\hline B3LYP-30 & 30 & 385 & 3.22 & 0.730 & 390 & 3.18 & 0.793 & 400 & 3.10 & 2.311 \\
\hline B3LYP & 20 & 434 & 2.86 & 0.589 & 439 & 2.82 & 0.643 & 440 & 2.82 & 1.837 \\
\hline PBE0 & 25 & 410 & 3.03 & 0.655 & 415 & 2.99 & 0.713 & 419 & 2.96 & 2.065 \\
\hline mPW1PBE & 25 & 410 & 3.03 & 0.655 & 415 & 2.99 & 0.713 & 419 & 2.96 & 2.067 \\
\hline CAM-B3LYP & 19 & 327 & 3.79 & 1.151 & 331 & 3.75 & 1.234 & 352 & 3.52 & 2.936 \\
\hline BMK & 42 & 355 & 3.49 & 0.898 & 360 & 3.45 & 0.971 & 374 & 3.32 & 2.673 \\
\hline$\omega \mathrm{B} 97 \mathrm{XD}$ & 22 & 315 & 3.93 & 1.314 & 318 & 3.89 & 1.403 & 343 & 3.62 & 3.081 \\
\hline M062X & 54 & 334 & 3.71 & 1.054 & 338 & 3.67 & 1.137 & 354 & 3.51 & 2.892 \\
\hline
\end{tabular}

${ }^{\text {a }}$ For the range-separated functionals the values correspond to the short-range exchange

${ }^{\mathrm{b}}$ Experimental data for $n$-hexane solution [6] 
Fig. 2 Frontier molecular orbitals - the highest occupied (HOMO) and the lowest unoccupied (LUMO) - of the studied dyes TR1-TR3
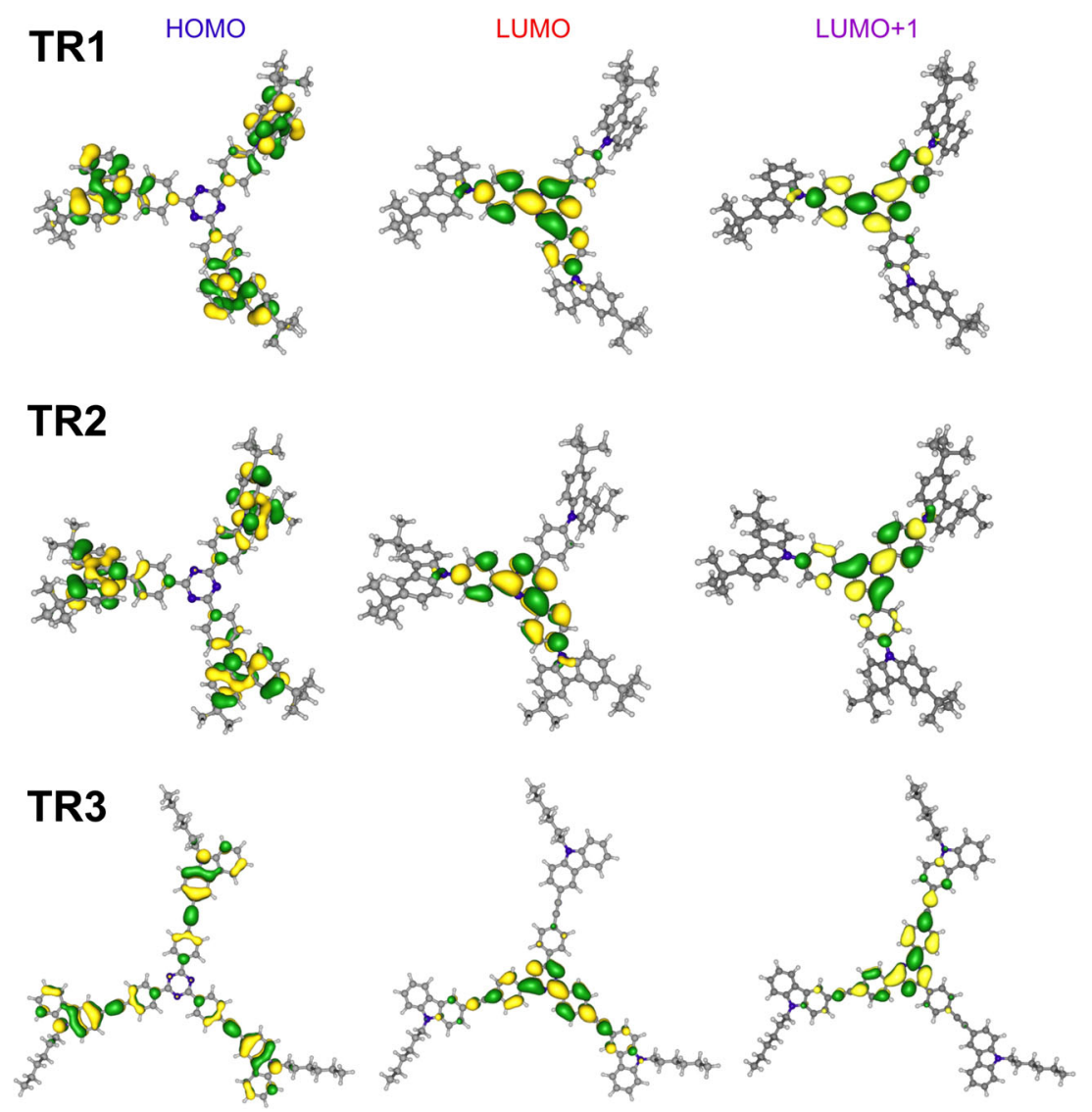

TR1-TR3 compounds can be recommended not only as emitters for OLEDs $[6,35,36]$ but also as light-harvesting materials for photovoltaic solar cells. Note that compounds TR1TR3 demonstrate high photoluminescence quantum yields reaching 0.85 [6].

\section{Nature of the absorption spectra}

In this section, we discuss the calculated data on the absorption spectra of TR1-TR3 molecules that are characterized by the close similar spectral properties (Table 2) due to the similar star-shaped structure (i.e., the same symmetry selection rules) and also the same D and A fragments for each of the three molecules. In Table 1, we selected only transitions with oscillator strengths $>0.01$. The first long-wavelength most intense absorption band for TR1-TR3 molecules corresponds to the quasi-degenerate electronic transitions to the $S_{1}$ and $S_{2}$ states; the main configurations for both $S_{1}$ and $S_{2}$ states are the $\mathrm{HOMO} \rightarrow \mathrm{LUMO}+1$ and $\mathrm{HOMO} \rightarrow$ LUMO, respectively. Thus, here we account for the fact that LUMO and LUMO+ 1 are almost degenerate. The HOMO, LUMO and LUMO+1 wavefunctions for the TR1-TR3 dyes are illustrated in Fig. 2, and the complete set of MOs involved in the electron transitions are presented in Figs. S3-S5.

As one can see in Fig. 2, the HOMO orbital for TR1-TR3 molecules is localized mainly on the carbazole moieties.
Table 2 Calculated wave lengths (eV), oscillator strength $(f)$ and orbital assignment for the quasi-degenerate $\mathrm{S}_{0} \rightarrow \mathrm{S}_{1}$ and $\mathrm{S}_{0} \rightarrow \mathrm{S}_{2}$ electronic transitions of TR1-TR3 molecules by the B3LYP-30/6-31G(d,p) method within IEFPCM approach ( $n$-hexane)

\begin{tabular}{|c|c|c|c|}
\hline Transition & $E(\mathrm{eV})$ & $f$ & Assignment \\
\hline \multicolumn{4}{|l|}{ TR1 } \\
\hline$S_{1}$ & 3.22 & 0.730 & $\begin{array}{l}\text { HOMO } \rightarrow \text { LUMO+1 }(+48 \%) \\
\text { HOMO-1 } \rightarrow \text { LUMO }(23 \%) \\
\text { HOMO-2 } \rightarrow \text { LUMO+1 }(20 \%)\end{array}$ \\
\hline$S_{2}$ & 3.22 & 0.731 & $\begin{array}{l}\text { HOMO } \rightarrow \text { LUMO }(+44 \%) \\
\text { HOMO }-2 \rightarrow \text { LUMO }(+24 \%) \\
\text { HOMO }-1 \rightarrow \text { LUMO+1 }(23 \%)\end{array}$ \\
\hline \multicolumn{4}{|l|}{ TR2 } \\
\hline$S_{1}$ & 3.18 & 0.793 & $\begin{array}{l}\text { HOMO } \rightarrow \text { LUMO+1 }(+50 \%) \\
\text { HOMO }-2 \rightarrow \text { LUMO }(+18 \%) \\
\text { HOMO }-1 \rightarrow \text { LUMO }+1(+17 \%)\end{array}$ \\
\hline$S_{2}$ & 3.18 & 0.794 & $\begin{array}{l}\text { HOMO } \rightarrow \text { LUMO }(+45 \%) \\
\text { HOMO-1 } \rightarrow \text { LUMO }(21 \%) \\
\text { HOMO }-2 \rightarrow \text { LUMO }+1(+19 \%)\end{array}$ \\
\hline \multicolumn{4}{|l|}{ TR3 } \\
\hline$S_{1}$ & 3.10 & 2.311 & $\begin{array}{l}\text { HOMO } \rightarrow \text { LUMO+1 }(+40 \%) \\
\text { HOMO-1 } \rightarrow \text { LUMO }(21 \%) \\
\text { HOMO-2 } \rightarrow \text { LUMO+1 }(20 \%)\end{array}$ \\
\hline$S_{2}$ & 3.10 & 2.311 & $\begin{array}{l}\text { HOMO } \rightarrow \text { LUMO }(+39 \%) \\
\text { HOMO }-2 \rightarrow \text { LUMO }(+21 \%) \\
\text { HOMO }-1 \rightarrow \text { LUMO+1 }(21 \%)\end{array}$ \\
\hline
\end{tabular}


Meanwhile, the LUMOs display electron densities primarily on the triazine core, and also on the phenyl bridges. Actually, both $S_{1}$ and $S_{2}$ states are characterized by their CT nature, but, at the same time, both HOMO and LUMO/LUMO+1 orbitals make large contributions to the common atoms of the linker (phenyl ring in the case of TR1, TR2 molecules and phenyl-ethynyl fragment in TR3). This provides a large transition dipole moment for the $S_{0}-S_{1}$ and $S_{0}-S_{2}$ transitions. In fact, the larger the common area for the HOMO and LUMO/LUMO+1 wavefunction, the higher the transition dipole moment and its oscillator strength. It can be seen from Table 2 that $S_{0}-S_{1}$ and $S_{0}-S_{2}$ transitions for the TR1 and TR2 molecules are characterized by almost the same oscillator strength values due to the same linker fragment. For the phenyl-ethynyl containing TR3 compound, the intensity of the $S_{0}-S_{1}$ and $S_{0}-S_{2}$ transitions is three times higher than for the TR1 and TR2 compounds due to longer linker fragment in the TR3 molecule. This fact is in good agreement with the experimental spectra regarding the first absorption band $\left(\varepsilon_{\max }^{0-0}=7 \times 10^{4} \mathrm{M}^{-1} \mathrm{~cm}^{-1}\right.$ for the TR1 and TR2 compounds, while for the TR3 compound, $\varepsilon_{\max }^{0-0}=$ $3.4 \times 10^{5} \mathrm{M}^{-1} \mathrm{~cm}^{-1}$ ) [6].

We should stress that the experimental absorption spectrum of TR1 (Fig. 3) exhibits three blue-shifted bands that are not reproduced by the vertical TD-DFT calculation. This is a progression of $\mathrm{C}-\mathrm{C}$ vibrations $\left(\nu=1276 \mathrm{~cm}^{-1}\right)$, which correspond mostly to the triazine-phenyl link. Upon HOMOLUMO excitation, this $\mathrm{C}-\mathrm{C}$ bond becomes much stronger. The HOMO is a non-bonding orbital with respect to such a link, but the LUMO is a bonding orbital (Fig. 3); thus, the force constant of this mode is higher in the excited state. Such a strong change in the force field, and of the mode displacement upon excitation, leads to the occurrence of a long progression of the $v_{\mathrm{C}-\mathrm{C}}$ mode $\left(1276 \mathrm{~cm}^{-1}\right)$ in the first absorption band. The higher energy band in the absorption spectrum of TR1 (at $4.16 \mathrm{eV}$ ), TR2 (at $4.13 \mathrm{eV}$ ) and TR3 (at $4.07 \mathrm{eV}$ ) corresponds to a local excitation of the $\pi \pi^{*}$-type in the carbazole moieties (Tables S3, S4).

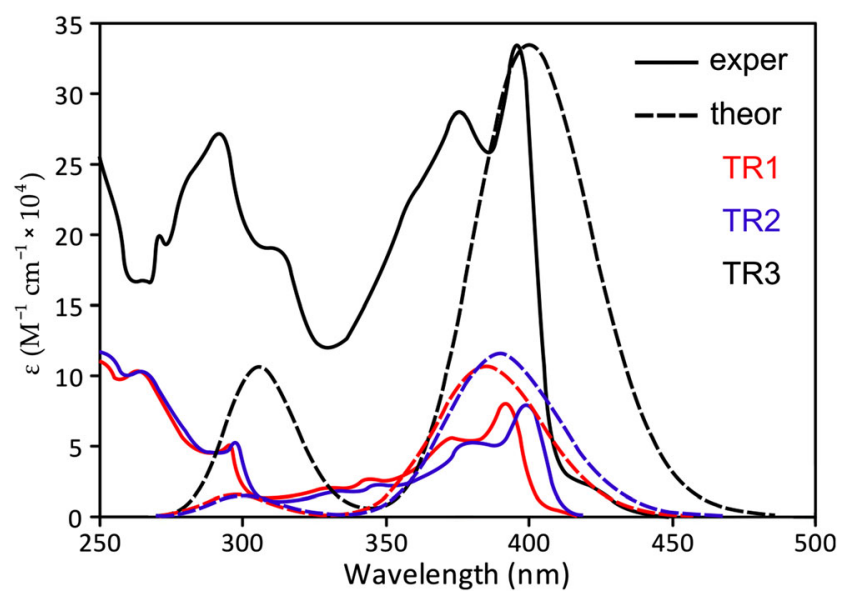

Fig. 3 Plot of the calculated absorption spectra of the studied dyes TR1TR3

\section{Solvatochromic effect}

An interesting experimental observation is that molecules TR1-TR3 demonstrate a strong solvatochromic effect only in the fluorescence spectra, but not in the absorption spectra. The authors of a previous study [6] explained this fact by the strong differences in electronic structure of the ground and first singlet excited states for the studied TR1-TR3 molecules. Therefore, we tracked the effect of solvent on molecular features such as permanent dipole moment of $\mathrm{S}_{0}$ and $S_{1}$ electronic states, and also on the energy of $S_{1}$ state, since the latter has a CT nature. The calculated data of the dipole moment dependence are presented in Fig. 4. Despite the symmetrical core of the molecular graph $\odot_{3}$ symmetry point group), the presence of carbazole ligands causes occurrence of a weak permanent dipole moment in the ground state of TR1-TR3 molecules (Fig. 4).

The corresponding dipole moment vector is oriented along the main molecular axis perpendicular to the triazine core plain (Fig. 5). As one can see in Fig. 4, the values of the ground state dipole moment $(\mu)$ are relatively small, and varying in the range of $0.1-1.0 \mathrm{D}$. This is because of the quasi- $C_{3}$ symmetry, which almost excludes the existence of a permanent dipole moment for the TR1-TR3 molecules in the ground state. As expected, the dipole moment values correlate with the dielectric permittivity of the solvents (Fig. 4). With a rise in solvent polarity, the $\mu$ value reaches a maximum in the

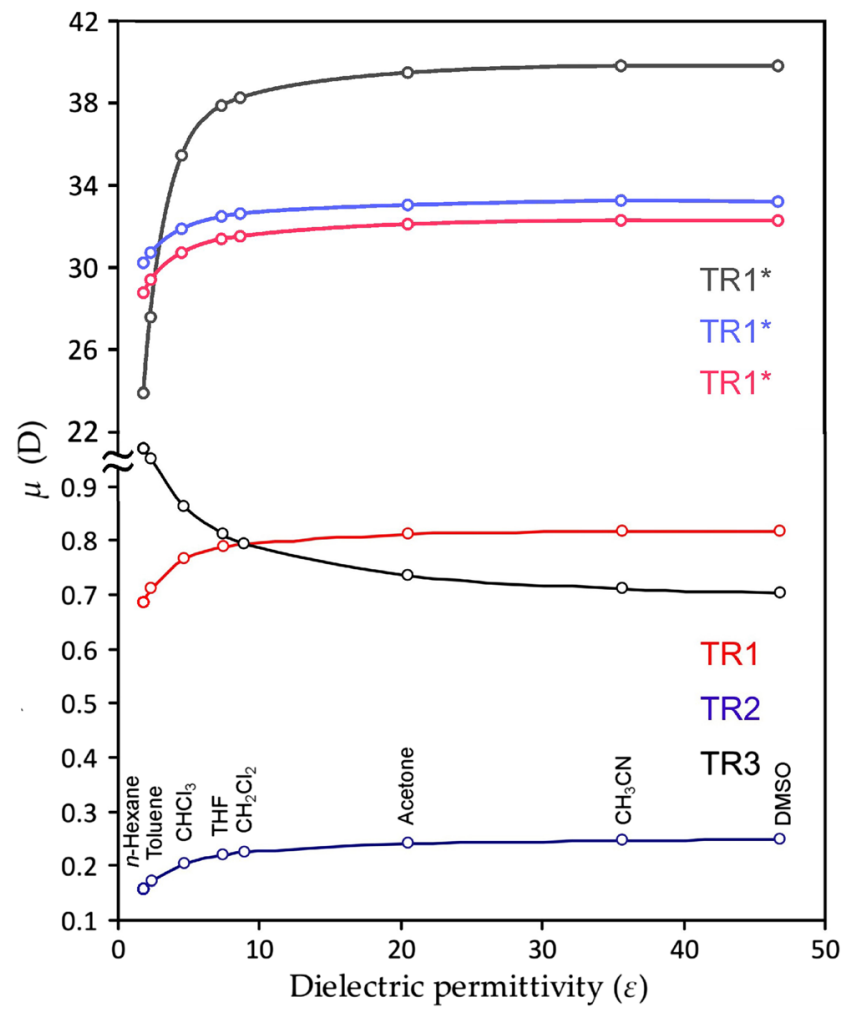

Fig. 4 Dipole moment $\mu$ (D) for the ground $\left(\mathrm{S}_{0}\right)$ and first excited $\left(\mathrm{S}_{1}, *\right)$ states of the TR1-TR3 dyes as a function of dielectric permittivity of solvent 


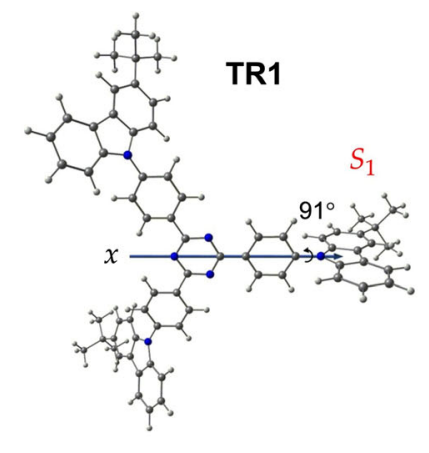

TR2
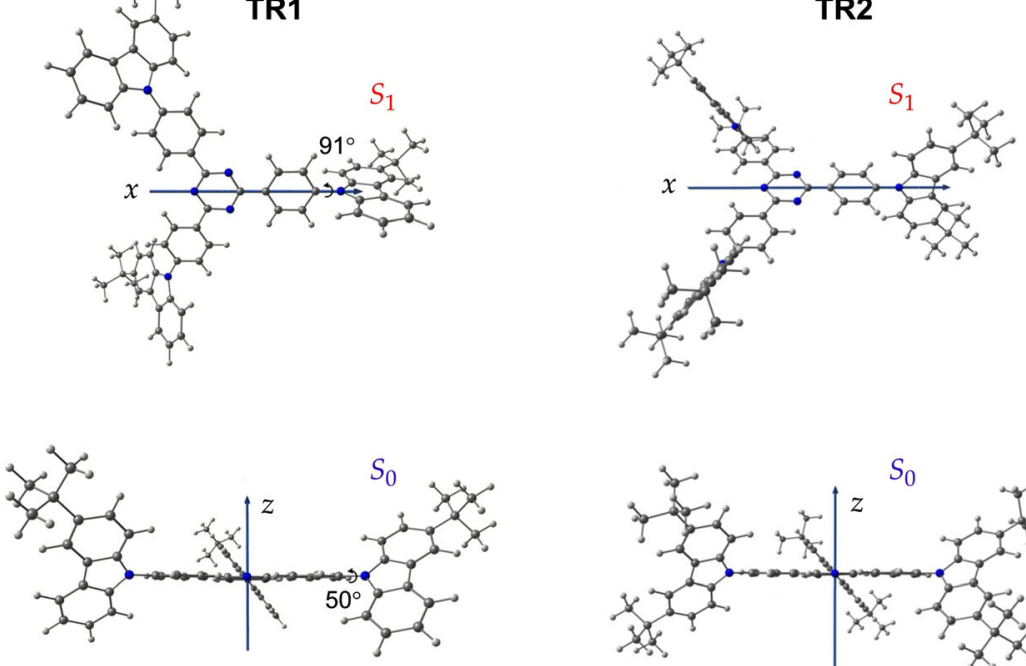

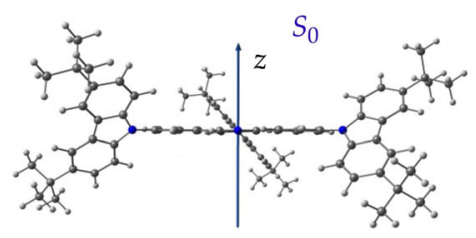

TR3

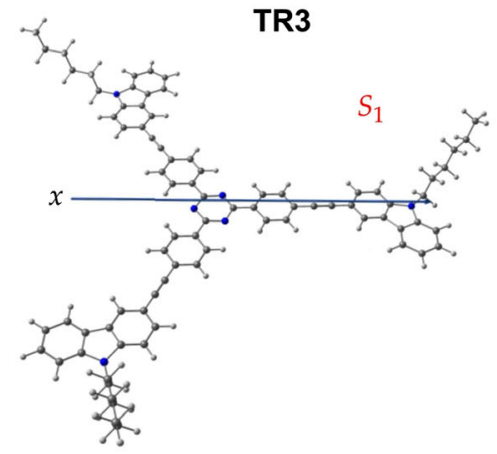

Fig. 5 Orientation of the permanent dipole moment vector for the ground $\left(S_{0}\right)$ and first excited $\left(S_{1}\right)$ singlet state of molecules TR1-TR3

case of TR1 and TR2. At the same time, the corresponding $\mu$ values for TR3 tend towards a minimum (Fig. 4). But, in all cases, the variation in the $\mu$ value is not more than $0.25 \mathrm{D}$ between the non-polar cyclohexane solvent and the strongly polar acetonitrile. A similar correlation occurs for the $S_{0}-S_{1}$ vertical transition energies vs. dielectric permittivity. Both the theoretical and experimental energies decrease towards the rise in solvent polarity (Table 3 ). The calculated transition energies, however, are more robust to a change of solvent; in practice, this effect is much more pronounced [6].

The $\mu$ value and vector orientation change crucially upon electronic excitation of TR1-TR3 molecules into the $S_{1}$ state (Figs. 4, 5). We predicted an increase of $>30$ times the permanent dipole moment for the $S_{1}$ exited state of molecules TR1TR3, which is caused by the strong charge separation upon the $S_{0}-S_{1}$ electronic transition of CT nature.
A strong electric polarization of the TR1-TR3 molecules in the $S_{1}$ excited state provides strong stabilization of the excited molecule in polar solvents in comparison with nonpolar media. This means that more polar solvents provide stronger solvation of the excited-state molecule, which leads to a decrease in $S_{1}$ state energy. This statement is in perfect qualitative agreement with experimental observations: the fluorescence wavelength increases strongly with the rise of solvent polarity. At the same time, the energy of the vertical $S_{0} \rightarrow S_{1}$ transition (in absorption) is almost insensitive for the solvent effect, which means a strong increase in Stokes shift $(\Delta v)$ with the rise in solvent polarity (experimentally, the $\Delta v$ values change from the $615 \mathrm{~cm}^{-1}$ in nonpolar hexane to $9842 \mathrm{~cm}^{-1}$ in highly polar acetonitrile). Though our IEFPCM-B3LYP-30/6$31 \mathrm{G}(\mathrm{d})$ calculations strongly overestimate quantitatively the solvation energy of the $S_{1}$ state of TR1-TR3, the overall

Table 3 The vertical (vert.) and adiabatic (ad.) $S_{0}-S_{1}$ transition energies (eV) for the TR1-TR3 molecules as a function of dielectric permittivity of solvent (theoretical values calculated using the B3LYP-30 scheme)

\begin{tabular}{|c|c|c|c|c|c|c|c|c|c|c|c|c|c|c|}
\hline \multirow[t]{2}{*}{ Dye } & \multicolumn{2}{|c|}{$n$-Hexane $(\varepsilon=1.88)$} & \multicolumn{2}{|c|}{ Toluene $(\varepsilon=2.37)$} & \multicolumn{2}{|c|}{$\mathrm{CHCl}_{3}(\varepsilon=4.71)$} & \multicolumn{2}{|c|}{$\mathrm{THF}(\varepsilon=7.43)$} & \multicolumn{2}{|c|}{$\mathrm{CH}_{2} \mathrm{Cl}_{2}(\varepsilon=8.93)$} & \multicolumn{2}{|c|}{ Acetone $(\varepsilon=20.49)$} & \multicolumn{2}{|c|}{$\mathrm{CH}_{3} \mathrm{CN}(\varepsilon=35.69)$} \\
\hline & Exp & Theor & Exp & Theor & Exp & Theor & Exp & Theor & Exp & Theor & Exp & Theor & Exp & Theor \\
\hline TR1 (vert.) & 3.12 & 3.22 & 3.22 & 3.18 & 3.27 & 3.19 & 3.28 & 3.2 & 3.3 & 3.2 & 3.33 & 3.2 & 3.35 & 3.2 \\
\hline TR1 (ad.) & 3.19 & 2.17 & 2.86 & 2.01 & 2.58 & 1.64 & 2.55 & 1.49 & 2.48 & 1.44 & 2.38 & 1.29 & 2.26 & 1.24 \\
\hline TR2 (vert.) & 3.12 & 3.18 & 3.15 & 3.18 & 3.2 & 3.19 & 3.24 & 3.2 & 3.24 & 3.2 & 3.26 & 3.2 & 3.35 & 3.2 \\
\hline TR2 (ad.) & 3.05 & 2.05 & 2.79 & 1.9 & 2.53 & 1.55 & 2.52 & 1.4 & 2.42 & 1.36 & 2.32 & 1.22 & 2.13 & 1.17 \\
\hline TR3 (vert.) & 3.15 & 3.1 & 3.15 & 3.05 & 3.18 & 3.06 & 3.17 & 3.05 & 3.19 & 3.05 & 3.23 & 3.05 & 3.24 & 3.05 \\
\hline TR3 (ad.) & 3.1 & 2.79 & 2.74 & 2.68 & 2.54 & 2.37 & 2.45 & 2.23 & 2.4 & 2.18 & 2.23 & 2.06 & 2.13 & 2.02 \\
\hline
\end{tabular}


tendency of the $S_{1}$ energy vs. solvent polarity dependence is in good agreement with the experimental data.

It is interesting to note that the conformational structure of the excited state TR1 and TR2 molecules is almost the same as the ground state structure, except for the dihedral angle between the triazine core and one of the carbazole moiety ( $50^{\circ}$ in $S_{0}$ vs. $90^{\circ}$ in the $S_{1}$ state). Such symmetry distortion is a clear manifestation of the Jahn-Teller effect for the quasidegenerate $S_{1}$ and $S_{2}$ states, which should be strictly degenerate within the strict $C_{3}$ symmetry point group constraints. For the TR3 molecule, the excited state conformational structure is the same as the ground state structure

\section{Conclusions}

In this work, we have presented a computational study of the absorption spectra for a series of star-shaped compounds containing both $\mathrm{D}$ (carbazole) and A (2,4,6-triphenyl-1,3,5-triazine) moieties bound through various linking bridges. These compounds demonstrate solvent-sensitive absorption in the whole visible range depending on solvent polarity. This is due to the strong charge-polarization of the studied molecules upon excitation into the $S_{1}$ excited state of CT nature. It has been confirmed by the very high permanent dipole moment for the $S_{1}$ excited state ( $>30 \mathrm{D}$ ) rather than for the $S_{0}$ state $(\leq 1 \mathrm{D})$. As a result, the solvatochromic effect should be observed only in fluorescence spectra, but not in absorption spectra, in complete agreement with experimental data.

An interesting feature of the $S_{0}-S_{1}$ transition for the studied compounds is a high intensity in the absorption spectrum, which is unusual for the CT transition. This result can be explained by the fact that the corresponding HOMO and LUMO+ 1 orbitals possess large contributions at the common atoms of the linker, which provides a large transition dipole moment for the $S_{0}-S_{1}$ transition. Actually, the solvent-dependent fluorescence for the studied compounds can be well explained by the localized CT excited state concept, which should be applicable to related D-A star-shaped systems.

The $\mathrm{C}_{3}$ symmetry point group for the studied systems determines that the $S_{1}$ and $S_{2}$ states are strictly degenerate in the vertical approximation. However, upon geometry relaxation in the excited state, these $S_{1}$ and $S_{2}$ states are split due to the Jahn-Teller effect. A study of this effect will be the subject of a future detailed investigation. Finally, we note that the studied star-shaped D-A compounds can be used to trigger color in OLEDs as controlled by the solvent.

Acknowledgments This work was supported by the Ministry of Education and Science of Ukraine, Research Fund (Grant No. 0113U001694). Computations were performed on resources provided by the Swedish National Infrastructure for Computing (SNIC) at the Parallel Computer Center (PDC) through the project "Multiphysics Modeling of Molecular Materials", SNIC 020/11-23.

Open Access This article is distributed under the terms of the Creative Commons Attribution 4.0 International License (http:// creativecommons.org/licenses/by/4.0/), which permits unrestricted use, distribution, and reproduction in any medium, provided you give appropriate credit to the original author(s) and the source, provide a link to the Creative Commons license, and indicate if changes were made.

\section{References}

1. Detert H, Lehmann M, Meier H (2010) Materials 3:3218-3330

2. Ren S, Zeng D, Zhong H, Wang Y, Qian S, Fang Q (2010) J Phys Chem B 114:10374-10383

3. Michaleviciute A, Gurskyte E, Volyniuk DYU, Cherpak VV, Sini G, Stakhira PY, Grazulevicius JV (2012) J Phys Chem C 116: 20769-20778

4. Kreger K, Bäte M, Neuber C, Schmidt H-W, Strohrieg P (2007) Adv Funct Mater 17:3456-3461

5. Jin R (2015) J Mol Model 21:219-228

6. Matulaitis T, Kostiv N, Grazulevicius JV, Peciulyte L, Simokaitiene J, Jankauskas V, Luszczynska B, Ulanski J (2016) Dyes Pigments 127:45-58

7. Hung W-Y, Chiang P-Y, Lin S-W, Tang W-C, Chen Y-T, Liu S-H, Chou P-T, Hung Y-T, Wong K-T (2016) ACS Appl Mater Interfaces 8:4811-4818

8. Cherpak V, Stakhira P, Minaev B, Baryshnikov G, Stromylo E, Helzhynskyy I, Chapran M, Volyniuk D, Hotra Z, Dabuliene A, Tomkeviciene A, Voznyak L, Grazulevicius JV (2015) ACS Appl Mater Interfaces 7:1219-1225

9. Angioni E, Chapran M, Ivaniuk K, Kostiv N, Cherpak V, Stakhira P, Lazauskas A, Tamulevičius S, Volyniuk D, Findlay NJ, Tuttle T, Grazulevicius JV, Skabara PJ (2016) J Mater Chem C 4:3851-3856

10. Baryshnikov GV, Minaev BF, Minaeva VA, Ning Z, Zhang Q (2012) Opt Spectrosc 112:168-174

11. Baryshnikov GV, Minaev BF, Myshenko EV, Minaeva VA (2013) Opt Spectrosc 115:484-490

12. Minaev BF, Baryshnikov GV, Minaeva VA (2011) Dyes Pigments 92:531-536

13. Baryshnikov GV, Minaev BF, Minaeva VA (2010) Opt Spectrosc 108:16-22

14. Baryshnikov GV, Minaev BF, Minaeva VA (2011) Opt Spectrosc 110:216-223

15. Minaev BF, Baryshnikov GV, Slepets AA (2012) Opt Spectrosc 112:829-835

16. Ooyama Y, Oda Y, Mizumo T, Harima Y, Ohshita J (2013) Eur J Org Chem 4533-4538

17. Baheti A, Singh P, Lee C-P, Thomas JKR, Ho K-C (2011) J Org Chem 76:4910-4920

18. Frisch MJ et al (2009) GAussian09, Revision A.02. Gaussian Inc, Wallingford

19. Kohn W, Sham L (1965) Phys Rev A 140:A1133-A1138

20. Becke AD (1993) J Chem Phys 98:5648-5652

21. Lee C, Yang W, Parr RG (1988) Phys Rev B 37:785-789

22. Hehre WJ, Radom L, Schleyer PVR, Pople JA (1986) Ab initio molecular orbital theory. Wiley, New York

23. Krishnan R, Binkley JS, Seeger R, Pople JA (1980) J Chem Phys $72: 650-654$ 
24. Stratmann RE, Scuseria GE, Frisch MJ (1998) J Chem Phys 109: $8218-8224$

25. Adamo C, Barone V (1999) J Chem Phys 110:6158-6170

26. Adamo C, Barone V (1998) J Chem Phys 108:664-675

27. Yanai T, Tew D, Handy N (2004) Chem Phys Lett 393:51-57

28. Boese AD, Martin JML (2004) J Chem Phys 121:3405-3416

29. Chai J-D, Head-Gordon M (2008) Phys Chem Chem Phys 10: 6615-6620

30. Zhao Y, Truhlar DG (2008) Theor Chem Accounts 120:215-241

31. Miertuš S, Scrocco E, Tomasi J (1981) Chem Phys 55:117-129
32. Gorelsky SI (2013) SWizard program. University of Ottawa, Ottawa, http://www.sg-chem.net/

33. Zhurko GA (2013) ChemCraft, version 1.6 (build 310). http://www. chemcraftprog.com/

34. Baryshnikov GV, Minaev BF, Slepets AA, Minaeva VA (2014) Opt Spectrosc 116:431-437

35. Wang Y, Liu W, Deng J, Xie G, Liao Y, Qu Z, Tan H, Liu Y, Zhu W (2016) Chem Asian J 11:2555-2558

36. Lv X, Wang B, Pan B, Huang Z, Xiang S, Tan J, Yi W, Huang H, Wang L (2016) RSC Adv 6:46775-46784 